\title{
Detection and Description of Spatial Patterns of Bacterial Brown Spot of Snap Beans Using Cyclic Samples
}

\author{
B. D. Hudelson, M. K. Clayton, K. P. Smith, and C. D. Upper
}

First, second, third, and fourth authors: Department of Plant Pathology; second author: Department of Statistics; fourth author: Agricultural Research Service, U.S. Department of Agriculture, University of Wisconsin, Madison 53706. Accepted for publication 30 September 1996.

\begin{abstract}
Hudelson, B. D., Clayton, M. K., Smith, K. P., and Upper, C. D. 1997. Detection and description of spatial patterns of bacterial brown spot of snap beans using cyclic samples. Phytopathology 87:33-41.

Snap bean plants within seven-row segments that ranged from 65 to $147 \mathrm{~m}$ were sampled, using a cyclic sampling plan. In the cyclic sampling plan, only 6 of every 31 plants were sampled, but sampled plants were spaced such that pairs of plants that were $1,2,3,4, \ldots, 1,525$ plants apart could be identified within each sample. Every leaflet on every sampled plant was assessed for bacterial brown spot, and the proportion of disease leaflets per plant was determined. Arcsine square-root-trans-
\end{abstract}

ABSTRACT formed disease incidence values were analyzed for spatial patterns by autocorrelation and spectral analyses. Disease patterns were detected at several different scales within a single snap bean row, at distances that ranged from $\sim 20$ to $\sim 100 \mathrm{~m}$. Approximately 23 to $53 \%$ of the disease variability in the samples could be described by sine and cosine curves, indicating a substantial component of regularity in the disease patterns. Possible origins for these regular patterns, including cultural practices and seed infestation, are discussed.

Additional keywords: adaptive sampling, Pseudomonas syringae.
In previous work $(16,17)$, we described the development of a sampling strategy for the detection and description of multiple spatial patterns of plant disease at several scales within a single field. Prior to our work, there had been a limited number of reports indicating the presence of such multiple disease patterns $(19,21,31)$. Our work was the first to propose a general strategy for systematically detecting multiple spatial patterns at multiple scales and the first to detect such patterns with this strategy, using bacterial brown spot of snap beans (Phaseolus vulgaris L.), caused by Pseudomonas syringae pv. syringae, as a model system.

Our sampling strategy, called adaptive sampling, involves the iterative application of two basic steps:

Step 1: Sampling and description of spatial patterns within an area of defined size in a field.

Step 2: Use of spatial-pattern information obtained in step 1 to develop new sampling plans for larger areas in the field.

These steps are quite general, and the exact details of sampling and data description can be tailored to accommodate the challenges of a particular crop or disease and to accommodate a variety of analytical approaches.

In step 1 of the first iteration of adaptive sampling in our own work, we looked for patterns of bacterial brown spot in short $(\sim 5 \mathrm{~m})$ row segments, using a sampling approach in which all plants within the row segment were assessed for disease (i.e., a total census was con-

Corresponding author: B. D. Hudelson; E-mail address: bdh@plantpath.wisc.edu

Mention of a trademark, proprietary product, or vendor does not constitute a guarantee or warranty of the product by the U.S. Department of Agriculture and does not imply its approval to the exclusion of other products or vendors that may also be suitable.

Publication no. P-1996-1122-01R

This article is in the public domain and not copyrightable. It may be freely reprinted with customary crediting of the source. The American Phytopathological Society, 1997. ducted). There are a variety of statistical tools available for describing data of this type, such as distance-class $(10,11,26,32)$ and geostatistical techniques, including kriging (8). These techniques have been used for the detection and description of plant disease spatial patterns $(5,14,18,20,22,23,27,28)$. For our work, we chose ARIMA modeling as a descriptive tool-a technique adapted from the statistical time series literature $(2-4,9)$ and a variation of which has been previously discussed in the context of describing plant disease spatial patterns $(24,25)$. In the context of adaptive sampling, ARIMA modeling allows for simultaneous detection of multiple spatial patterns within a single data set and provides a quantification of these patterns. In addition, the statistical theory underlying ARIMA modeling is quite powerful and sufficiently well developed that analytical tools are available to assess the goodness-of-fit of such models. The underlying statistical theory for ARIMA models also is sufficiently sophisticated that information from the models can be easily incorporated into existing statistical sampling theory to aid in the development of sampling plans in step 2 of adaptive sampling.

Using ARIMA modeling, we were able to detect nonrandom disease variability at two scales in our total-census data $(16,17)$. The first of these patterns was a positive correlation between disease incidence on adjacent plants, corresponding to a slowly undulating change in disease levels within a row segment. A second pattern of smaller scale and magnitude that gave the dominant undulating pattern a jagged appearance also was detected. In more than $90 \%$ of the total-census samples we collected, we found that bacterial brown spot variability could be described by a generalized ARIMA(ll 0 1) model (16,17):

$$
Y_{t}=\phi_{1} Y_{t-1}+\varepsilon_{t}-\theta_{1} \varepsilon_{t-1}+\delta
$$

with $0 \leq \phi_{1} \leq 1$ and $0 \leq \theta_{1} \leq \phi_{1}$. In this model, $Y_{t}$ and $Y_{t-1}$ are arcsine square-root-transformed proportions of diseased leaflets associated with the plants at positions $t$ and $t-1$ in the row. The terms $\varepsilon_{t}$ and $\varepsilon_{t-1}$ are random errors (with a variance of $\sigma_{\varepsilon}^{2}$ ) associated with these plants. The terms $\phi_{1}$ and $\theta_{1}$ are constants that quantify the two scales in disease patterns we observed, and $\delta$ is a constant related to the mean level of disease in the row segment. 
The sampling plan we developed based on our initial ARIMA modeling, for use in step 1 of the second iteration of adaptive sampling, was the 2,4-systematic sample, a special case of a more general class of samples, called $A, B$-systematic samples (17). In 2,4-systematic sampling, 2 adjacent snap bean plants are assessed for bacterial brown spot, the next 4 adjacent plants are skipped, the next 2 adjacent plants are assessed, etc. Disease incidence values from each group of two assessed plants (suitably transformed where appropriate) are averaged, and these averages are modeled using ARIMA models. Using this sampling plan, we were able to detect not only the two disease patterns detected in the first iteration of adaptive sampling, but an additional pattern characterized by correlations in disease for plants that were 6 to 24 plants apart (17). The ARIMA models fit to 2,4-systematic sample data also provided information for developing new sampling plans to use in further iterations of adaptive sampling.

2,4-systematic sampling (and $A, B$-systematic sampling in general) is not without drawbacks, however. 2,4-systematic samples condense information, so patterns that occur, for example, every 18 plants are difficult, if not impossible, to distinguish from patterns that occur every 23 plants. The regular spacing of pairs of sampled plants appears to be one reason for this ambiguity. Within a 2,4-systematic sample, pairs of plants that are 1, 5, 6, 7, 11, 13, 17,18 , etc. plants apart can be identified. However, plant pairs separated by other distances are missing. Thus, the disease information associated with these missing distances also is absent. A more desirable sampling plan would be one in which only a fraction of the plant population is evaluated for disease but in which all plant pair distances (and, thus, information about disease at all distances) are represented in the sample.

Clinger and Van Ness (7) described a series of systematic sampling plans, called cyclic samples, that fulfill these criteria. Their

TABLE 1. Characteristics of Wisconsin fields from which cyclic and totalcensus samples of bacterial brown spot of snap beans were collected during 1988 to 1989

\begin{tabular}{lllcl}
\hline $\begin{array}{l}\text { Field } \\
\text { identification } \\
\text { number }\end{array}$ & $\begin{array}{c}\text { Wisconsin } \\
\text { county }\end{array}$ & $\begin{array}{l}\text { Nearest } \\
\text { village }\end{array}$ & $\begin{array}{c}\text { Field area } \\
\text { (ha) }\end{array}$ & $\begin{array}{c}\text { Bean } \\
\text { cultivar }\end{array}$ \\
\hline 14 & Waushara & Almond & 4 & BBL 94 94 \\
16 & Columbia & Arlington & 8 & BBL 109 \\
17 & Green Lake & Markesan & 14 & Peak \\
19 & Green Lake & Fairwater & 31.2 & Peak \\
21 & Green Lake & Fairwater & 60 & Peak \\
\hline
\end{tabular}

a Bush Blue Lake.

TABLE 2. Summary of characteristics of cyclic and total-census samples of bacterial brown spot of snap beans

\begin{tabular}{|c|c|c|c|c|c|}
\hline \multirow[b]{2}{*}{$\begin{array}{l}\text { Date } \\
\text { collected }\end{array}$} & \multicolumn{2}{|c|}{ Cyclic sample } & \multicolumn{3}{|c|}{ Total-census sample } \\
\hline & Code $^{a}$ & $\begin{array}{l}\begin{array}{l}\text { Length } \\
(\mathrm{m})^{\mathrm{b}}\end{array}\end{array}$ & Code & $\begin{array}{l}\text { Length } \\
(\mathrm{m})\end{array}$ & $\begin{array}{l}\text { No. of } \\
\text { plants }\end{array}$ \\
\hline 23 Aug. 1988 & 14.C1 & 89.7 & 14.2 & 5.0 & 77 \\
\hline 27 Jul. 1989 & 16.C1 & 65.3 & 16.1 & $\mathrm{ND}^{\mathrm{c}}$ & 100 \\
\hline & & & 16.2 & ND & 102 \\
\hline 10 Aug. 1989 & 17.C1 & 108.2 & 17.2 & ND & 102 \\
\hline 24 Aug. 1989 & 17.C2 & 108.2 & & & \\
\hline 1 Sep. 1989 & 19.C1 & 105.1 & 19.1 & ND & 100 \\
\hline 22 Sep. 1989 & 21.C1 & 127.0 & 21.1 & ND & 101 \\
\hline 22 Sep. 1989 & 21.C2 & 146.4 & 21.2 & ND & 100 \\
\hline
\end{tabular}

a Numbers to the left of the decimal point refer to the snap bean field from which cyclic and total-census samples were collected. The use of the letter C plus a number to the right of the decimal point denotes a cyclic sample. Single digits to the right of the decimal point distinguish total-census samples collected from the same field. All total-census samples, except sample 14.2, were collected from the same row segments from which corresponding cyclic samples were collected.

${ }^{b}$ All bean row segments from which cyclic samples were collected had 1,550 plants.

${ }^{\mathrm{c}}$ Not determined. work described a means of calculating the minimum fraction of a population of fixed size that must be sampled for the sample to contain pairs of observations that are any arbitrary distance apart (e.g., one, two, three, etc. plants apart). In addition, for several population sizes they outlined the exact spacing of observations in cyclic samples that would be needed to ensure that all plant pair distances would be represented.

Once data have been collected by a cyclic sample, one can calculate a correlation coefficient for disease values associated with all pairs of plants that are one, two, three, etc. plants apart (6). The resulting correlation coefficients can be plotted as a function of the plant pair distances to obtain a sample autocorrelation function (ACF), a descriptive tool derived from statistical time series literature $(2-4,9)$ that also can be used for detecting spatial patterns. Clayton and Hudelson (6) extended the usefulness of cyclic sampling plans by deriving a means of calculating confidence intervals (CIs) for ACFs derived from cyclic sample data assuming particular underlying ARIMA models.

The work described here is the first application of the work of Clinger and Van Ness (7) and Clayton and Hudelson (6) in the study of plant disease epidemics, with the specific goal of elucidating spatial patterns of bacterial brown spot of snap beans in step 1 of the second iteration of adaptive sampling.

\section{MATERIALS AND METHODS}

Sample collection. Seven cyclic samples were collected in 1988 and 1989 from five commercial snap bean fields located in central and south-central Wisconsin. More detailed information on the characteristics (location, cultivar, area, etc.) of these fields is provided in Table 1. Detailed descriptions of cyclic and total-census samples collected from these fields are provided in Table 2.

Cyclic samples were collected from snap bean row segments that were 1,550 plants in length (65.3 to $146.4 \mathrm{~m})$. Within each row segment, except the segment yielding cyclic sample 21.C2, all plants were labeled sequentially with tags numbered 1 through 1,550. Tags were color coded, so plants to be assessed for bacterial brown spot could be easily distinguished. For data set 21.C2, only plants to be assessed were tagged. Cyclic samples 17.C1 and 17.C2 were assessments of the same plants on two different dates. Cyclic samples 21.C1 and 21.C2 were collected from adjacent rows in the same field.

The spacing of assessed plants is shown in Figure 1. This spacing is a modification of a spacing suggested by Clinger and Van Ness (7) and was used to collect all of the cyclic samples in this study. The starting point of the sample (i.e., the first plant sampled) was selected randomly. Although only 6 of every 31 plants were assessed for disease, spacing of assessed plants was such that pairs of plants that were $1,2,3,4, \ldots, 1,525$ plants apart could be identified within each sample.

In addition to cyclic samples, total-census samples from short row segments (77 to 102 plants) were collected from each field. Cyclic and total-census samples were collected from within the same snap bean row, except for samples 14.2 and 14.C1, which were collected from adjacent rows. Total-census samples provided information on small-scale spatial patterns, which was used to construct $95 \%$ CIs for ACFs calculated from cyclic sample data (discussed below).

Disease assessment. Leaflets on each sampled plant were assessed for bacterial brown spot as described previously (16). Cyclic samples 21.C1 and 21.C2 were each assessed by a single worker, cyclic samples 14.C1, 17.C1, 17.C2, and 19.C1 were each assessed by two workers, and data set 16.C1 was assessed by four workers. Total-census samples were collected by a single worker.

Data analysis. For total-census samples, the disease incidence of each sampled plant was expressed as the proportion of diseased leaflets on each plant. Proportions were arcsine square-root-transformed to stabilize variance (29) and analyzed by a two-sided runs test (13) and autocorrelation $(2-4,9)$ and ARIMA analyses $(2-4,9)$. 
Data from cyclic samples assessed by more than one worker (14.C1, 16.C1, 17.C1, 17.C2, and 19.C1) were standardized when necessary to take into account assessor differences. For cyclic sample 14.C1, the necessity for standardization was determined by comparing the mean disease estimates among assessors, using a $t$ test $(\alpha=0.05)$. In this cyclic sample, no statistically significant difference in means was detected, and thus, standardization was deemed unnecessary. For all other cyclic samples, the total number of leaflets and the number of diseased leaflets of a small sample of plants (10 to 20) evaluated by all assessors of a given cyclic sample were compared in separate regression analyses. The total or diseased leaflet numbers obtained by the most experienced of the assessors evaluating a given cyclic sample (denoted as assessor 1) were used as dependent variables. Total or diseased leaflet numbers obtained by other assessors (denoted as assessors 2, 3, and 4) were used as independent variables. Assessments of total or diseased leaflet numbers were considered different when the slope of the resulting regression line was significantly different from one or the intercept of the line was significantly different from $0(\alpha=0.05)$. If a slope or an intercept value was determined to be significantly different based on these criteria (as in samples 16.C1, 17.C2, and 19.C1), then the resulting regression equation was used to calculate the new total or diseased leaflet numbers for assessor 2 (and where appropriate assessors 3 and 4) standardized to assessor 1.

Once total and diseased leaflet numbers were standardized, the proportion of diseased leaflets of each plant was calculated. Values less than 0 were truncated to 0 , and values greater than 1 were truncated to 1 . The resulting proportions were arcsine square-root transformed, and transformed values were used in subsequent analyses.

Cyclic samples 17.C1 and 17.C2, assessments of the same plants on two different dates, were compared initially by regression analysis, using MINITAB, release 5.1.1 (Minitab, The Pennsylvania State University, University Park). Transformed disease values from cyclic sample 17.C1 served as the independent variable, and transformed disease values from cyclic sample 17.C2 served as the dependent variable. This analysis provided information on temporal variation in the row segment from which these two cyclic samples were collected.

For all cyclic samples, sample ACFs, $r(s)$, were calculated using the results of Clayton and Hudelson (6). In $r(s), s$ is a lag value expressed as a distance measured in numbers of plants. For the cyclic samples collected in this study, $r(s)$ was calculated for all $s$ : $1 \leq s \leq 700$.

An approximate $95 \%$ CI for each value of $\rho(s)$, the theoretical ACF that the sample ACF estimates, was determined using the results of Clayton and Hudelson (6). CIs were estimated based on the type of ARIMA model that best described the total-census data collected in conjunction with a given cyclic sample, typically

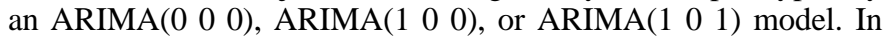
the special case in which an ARIMA(l $\left.\begin{array}{lll}0 & 1 & 1\end{array}\right)$ model best described the total-census data, direct calculation of CIs based on this model was not possible (the results of Clayton and Hudelson [6] are not applicable to models of this type). Therefore, two alternative $95 \%$ CIs for the ACF from the corresponding cyclic sample were calculated: a CI based on an underlying ARIMA( $\left.\begin{array}{lll}0 & 0 & 0\end{array}\right)$ model (a standard CI calculated for ACFs from data sets in which a priori information on spatial patterns is not available) and a CI based on the ARIMA(l $\left.\begin{array}{lll}1 & 0 & 1\end{array}\right)$ model that best fit the total-census data (used as an approximation of the ARIMA(l $\left.\begin{array}{lll}0 & 1 & 1\end{array}\right)$ model).

An excess number of values of $s$ for which $r(s)$ was outside the 95\% CI was considered evidence that disease patterns, in addition to those observed in the corresponding total-census data, were present in the row segment from which the cyclic sample was collected. Because a 95\% CI was used and 700 values of $r(s)$ were evaluated, we expected to observe, on average, 35 values of $s$ for which the values of $r(s)$ would be outside the CI $(3,4)$. Thus, an excess number of values of $s$ was defined as 36 or more. In addition, patterns in a sample ACF, for small values of $s(s \leq 50)$, inconsistent with expected patterns based on the ARIMA model fit to the corresponding total-census data set were considered evidence of additional spatial patterns of bacterial brown spot.

In addition to autocorrelation analysis, cyclic sample data were analyzed by a method similar to spectral analysis (2-4). Use of this analysis allowed us to determine whether there was evidence of regular spatial patterns that recurred at specific distances. In this analysis, a regression model of the form

$$
Y_{t}=\beta_{0}+\beta_{c, j} \cos \left(2 \pi t / \omega_{j}\right)+\beta_{s, j} \sin \left(2 \pi t / \omega_{j}\right)+a_{t}
$$

was fit to cyclic sample data. $Y_{t}$ is the assessor-standardized, arcsine square-root-transformed disease incidence value associated with the plant at position $t$ in the cyclic sample, $\beta_{c, j}, \beta_{s, j}$, and $\beta_{0}$

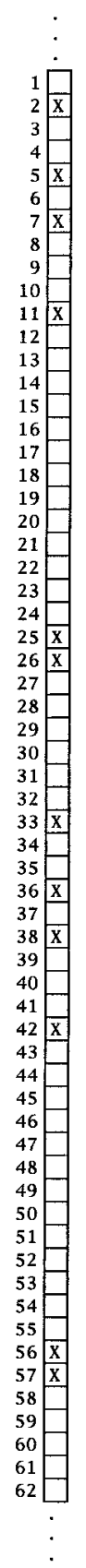

Fig. 1. Diagram of the cyclic sample used for field studies of the spatial variability of bacterial brown spot of snap beans. Each box represents a bean plant, and boxes with Xs represent the plants sampled and evaluated for disease. This cyclic sample repeated every 31 plants, and 6 of every 31 plants were sampled. The starting point of the sample (i.e., the first plant sampled) was randomly selected. Thus, the diagrammed cyclic sample is 1 of 31 possible cyclic samples. 
are the parameters of the regression equation, $\omega_{j}$ is a wavelength of the form $\omega_{j}=j(j=2,3,4, \ldots, 1,550)$, and $a_{t}$ is a random error term (with a variance of $\sigma_{a}^{2}$ ). Parameters were estimated by ordinary least squares. From each regression analysis (1,449 total), a multiple correlation coefficient, $R^{2}$, was obtained, indicating the proportion of the variability in the data that was explained by sine and cosine curves of a particular wavelength. $R^{2}$ values were plotted as a function of wavelength, and wavelengths associated with peaks in $R^{2}$ that extended beyond a $95 \%$ CI were considered wavelengths at which additional patterns of brown spot occurred.

The $95 \%$ CIs for $R^{2}$ plots were obtained based on information from ARIMA models that best fit total-census data collected in conjunction with cyclic samples. The expected values of $R^{2}, \rho^{2}$, were calculated by these models (29). Values of $\rho^{2}$ were $\tanh ^{-1}$ square-root transformed (29) to yield $Z$ values that were approximately normally distributed with $E(Z)=\tanh ^{-1}(\rho)$ and $V(Z)=1 /(n-$ $3)$, where $n$ is the number of sampled plants in the cyclic sample. A one-sided $95 \%$ CI for $E(Z)$ was calculated as $E(Z)+Z_{\alpha} /(n-$ $3)^{1 / 2}$, where $Z_{\alpha}$ is the appropriate value from a standard normal table. Two $Z_{\alpha}$ values, 1.645 and 3.9, were used. A $Z_{\alpha}$ value of 1.645 provided a "liberal" $95 \%$ CI. This CI does not take into account the multiple comparisons problem that arises from calculating CIs for a large number of wavelengths $(1,449)$. To account for multiple comparisons, a $Z_{\alpha}$ value of 3.9 was used to construct a Bonferroni-type 95\% CI (30)-a "conservative" 95\% CI. Once the CI (either liberal or conservative) for $E(Z)=\tanh ^{-1}(\rho)$ was determined, the CI for $\rho^{2}$ was obtained by applying the tanh transformation to $E(Z)+Z_{\alpha} /(n-3)^{1 / 2}$ and squaring the resulting $\rho$ value.

As a follow-up to spectral analysis, a stepwise regression procedure that alternated forward and backward steps (12) was used to select a subset of sine and cosine curves that described the nonrandom disease variability in each cyclic sample. The criterion in each forward step for incorporating a sine and cosine curve (as a pair) of a particular wavelength into the model was that the $F$ statistic associated with these curves should be 3.02 or larger. This procedure was approximately equivalent to performing an $F$ test with 2 (the number of predictors in the regression) and 300 (the total number of observations in the cyclic sample) degrees of freedom and $\alpha=0.05$ of the null hypothesis that the sine and cosine curves were not needed in the model. Similarly, in each backward step, sine and cosine curves of a given wavelength were removed from the model if the associated $F$ statistic was less than 3.02. This procedure yielded a model of the form

$$
\begin{gathered}
Y_{t}=\beta_{0}+\beta_{s, 1} \sin \left(2 \pi t / \omega_{1}\right)+\beta_{c, 1} \cos \left(2 \pi t / \omega_{1}\right)+\ldots+ \\
\beta_{s, i} \sin \left(2 \pi t / \omega_{i}\right)+\beta_{c, i} \cos \left(2 \pi t / \omega_{i}\right)+e_{t}
\end{gathered}
$$

Here $\omega$ 's are the wavelength of sine and cosine curves selected by the stepwise regression procedure, $\beta$ 's are the regression coef- ficient that can be estimated using least squares, and $e_{t}$ is a random error term (with a variance of $\sigma_{e}^{2}$ ).

Data that could be modeled with sine and cosine curves with a wavelength of 1,550 plants were, as an alternative, also modeled with either linear or linear and quadratic trends. This analysis allowed us to consider the possibility that nonsinusoidal disease patterns might be present in the cyclic samples. Residuals from models with linear or linear and quadratic trends were analyzed by stepwise regression similar to that described above to identify sine and cosine curves that would describe the remaining nonrandom variability in the data. This led to a final model of

$$
\begin{gathered}
Y_{t}=\beta_{0}+\beta_{1} t+\beta_{s, 1} \sin \left(2 \pi t / \omega_{1}\right)+\beta_{c, 1} \cos \left(2 \pi t / \omega_{1}\right)+\ldots+ \\
\beta_{s, i} \sin \left(2 \pi t / \omega_{i}\right)+\beta_{c, i} \cos \left(2 \pi t / \omega_{i}\right)+e_{t}
\end{gathered}
$$

or

$$
\begin{gathered}
Y_{t}=\beta_{0}+\beta_{1} t+\beta_{2} t^{2}+\beta_{s, 1} \sin \left(2 \pi t / \omega_{1}\right)+\beta_{c, 1} \cos \left(2 \pi t / \omega_{1}\right)+\ldots+ \\
\beta_{s, i} \sin \left(2 \pi t / \omega_{i}\right)+\beta_{c, i} \cos \left(2 \pi t / \omega_{i}\right)+e_{t}
\end{gathered}
$$

\section{RESULTS}

Total-census data sets. Analyses of total-census data collected in conjunction with cyclic samples have been described previously (17). A summary is provided in Table 3. Runs analyses (13) indicated that three of the data sets $(16.1,17.2$, and 19.1) had more runs than expected, and three data sets had fewer runs than expected. However, the $P$ values for these runs tests were all greater than 0.05 , indicating the number of runs was not different from what one would expect due to chance. A runs test for data set 16.2 could not be performed because the median disease incidence for this data set was 0 .

Sample ACFs for four of the seven data sets $(14.2,16.1,16.2$, and 17.2) had no values of $r(s)$ outside corresponding 95\% CIs. These data could be modeled by an ARIMA( $\left(\begin{array}{lll}0 & 0 & 0\end{array}\right)$ model (a generalized ARIMA(1 011$)$ model with $\left.\phi_{1}=\theta_{1}=0\right)$. For two data sets (21.1 and 21.2), $r(1)$ was outside the corresponding 95\% CI, and these data sets were modeled by ARIMA( $\left.\begin{array}{lll}1 & 0 & 0\end{array}\right)$ models (a generalized ARIMA(llll $\left.\begin{array}{lll}1 & 0\end{array}\right)$ model with $\phi_{1} \neq 0$ and $\theta_{1}=0$ ). Finally, data set 19.1 had seven values of $r(s)$ outside the $95 \%$ CI and was modeled by an ARIMA( $\left.\begin{array}{lll}0 & 1 & 1\end{array}\right)$ model (a generalized ARIMA( $\left.\begin{array}{lll}1 & 0 & 1\end{array}\right)$ model with $\phi_{1}=1$ and $\left.\theta_{1} \neq 0\right)$. Parameter estimates for these models are given in Table 3 .

Cyclic samples: General description. Nontransformed disease incidence values (standardized for assessor differences) were extremely variable within cyclic samples (Fig. 2). In five of the seven data sets, disease incidence values ranged from 0 to $100 \%$. In

\begin{tabular}{|c|c|c|c|c|c|c|c|}
\hline Data set $^{\mathrm{a}}$ & $\begin{array}{c}\text { Mean } \\
\text { disease }(\%)\end{array}$ & $\begin{array}{c}\text { ARIMA } \\
\text { model }\end{array}$ & $\phi_{1}$ estimate $^{\mathrm{b}}$ & $\theta_{1}$ estimate $^{\mathrm{b}}$ & $\sigma_{\varepsilon}^{2}$ estimate $^{\mathrm{b}}$ & $\begin{array}{l}\text { Significant } \\
\text { ACF lags }\end{array}$ & $\begin{array}{c}\text { Runs test } \\
Z \text { value }^{\mathrm{d}}\end{array}$ \\
\hline 14.2 & 55.4 & 000 & $0(\mathrm{ND})$ & 0 (ND) & 0.028 & 9 & -0.70 \\
\hline 16.1 & 10.5 & 000 & 0 (ND) & 0 (ND) & 0.028 & NONE & +0.63 \\
\hline 16.2 & 7.8 & 000 & 0 (ND) & 0 (ND) & 0.018 & NONE & $\mathrm{CND}^{\mathrm{e}}$ \\
\hline 17.2 & 45.6 & 000 & 0 (ND) & 0 (ND) & 0.144 & NONE & +0.40 \\
\hline 19.1 & 37.3 & 011 & $1(\mathrm{ND})$ & $0.875(0.048)$ & 0.032 & $1,2,4-7,11$ & +0.20 \\
\hline 21.1 & 60.2 & 100 & $0.340(0.094)$ & 0 (ND) & 0.050 & 1 & -1.42 \\
\hline 21.2 & 69.8 & 100 & $0.260(0.098)$ & 0 (ND) & 0.048 & 1 & -1.81 \\
\hline
\end{tabular}
particular, data set 17.C1 (Fig. 2D1) exhibited a large number of plants that were either totally disease free or were diseased on every leaflet. Mean disease levels for the seven cyclic samples

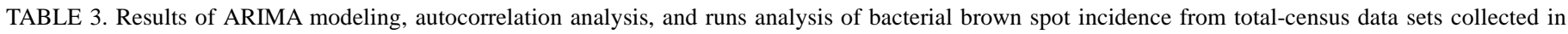
conjunction with cyclic samples

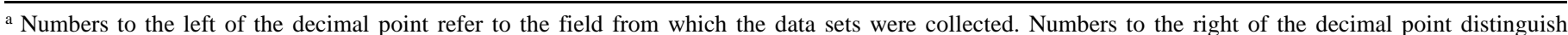
between data sets collected from the same field.

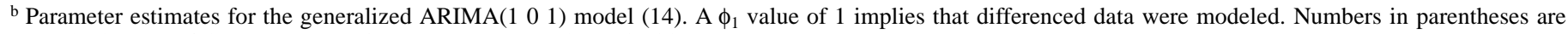
standard errors of the parameter estimates. (ND) $=$ Not determinable.

${ }^{\mathrm{c}}$ Autocorrelation function (ACF) lags among the first 20 lags for which autocorrelation function values lay outside a $95 \%$ confidence interval.

d None of the listed $Z$ values are statistically significant $(\alpha=0.05)$.

e Could not determine. Median disease was 0 . 
were $49.1,5.9,48.6,37.0,29.2,68.5$, and $52.2 \%$, respectively, for data sets 14.C1 (Fig. 2A), 16.C1 (Fig. 2C1), 17.C1 (Fig. 2D1), 17.C2 (Fig. 2E1), 19.C1 (Fig. 2B1), 21.C1 (Fig. 2F), and 21.C2 (Fig. 2G). Within a given cyclic sample, the mean disease level fluctuated from location to location in the row segment. This was particularly evident in cyclic sample 14.C1 (Fig. 2A). The level of disease in this data set was higher at plant numbers of approximately 40, 360, 790, and 1,275 and lower at plant numbers of approximately 290,650 , and 1,100 . The comparison of data sets 17.C1 and $17 . \mathrm{C} 2$ by regression indicated that $\sim 31 \%$ of the vari-
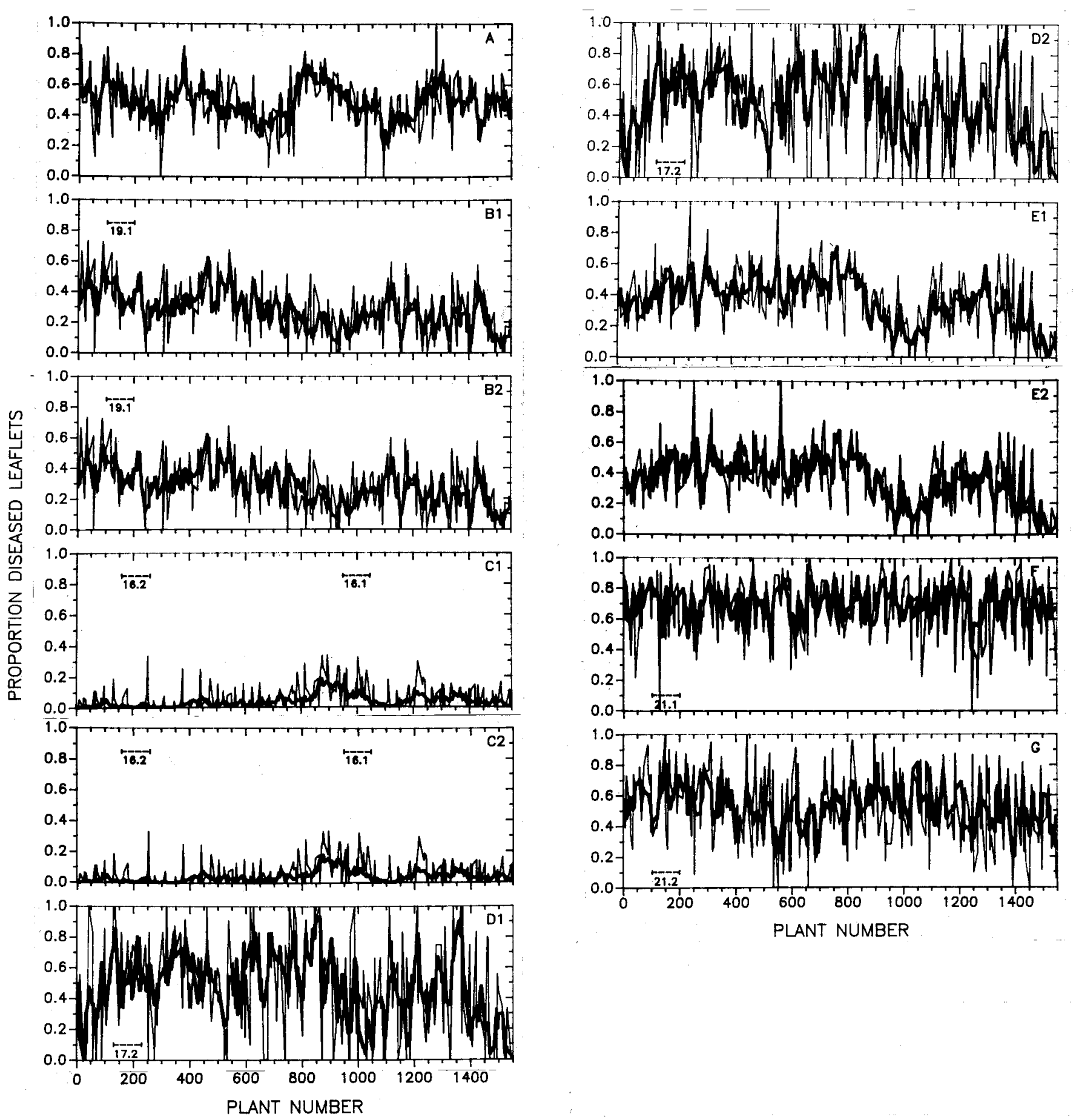

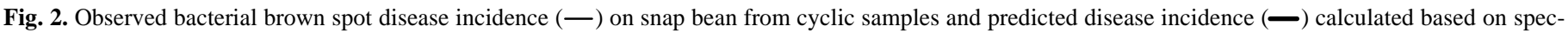

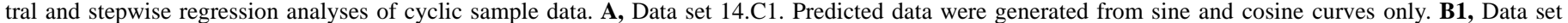

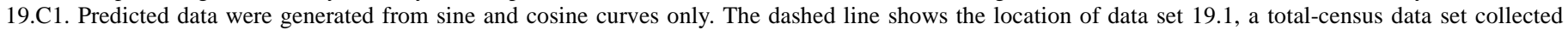

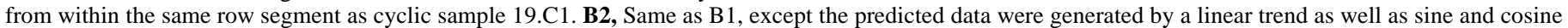

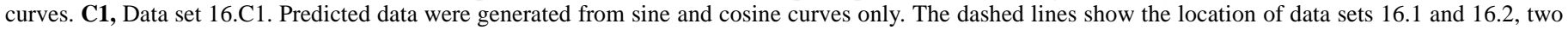

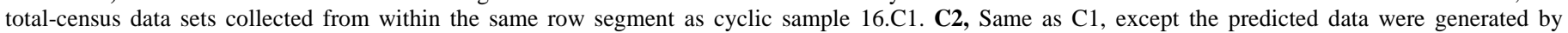

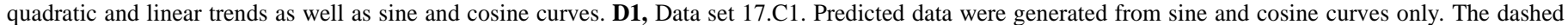

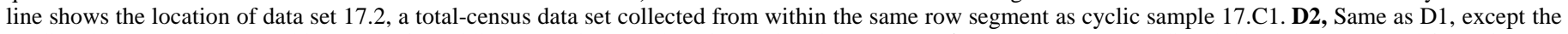

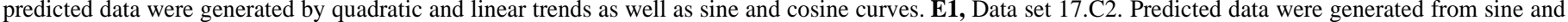

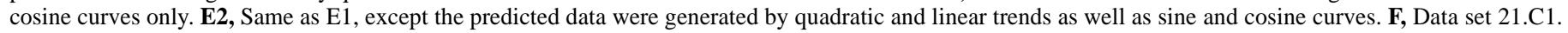

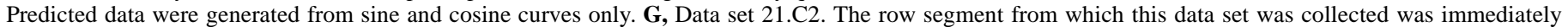
adjacent to the row segment from which the data in $\mathbf{F}$ was collected. Predicted data were generated from sine and cosine curves only. 
ability in disease in data set 17.C2 could be explained by the variability in disease in data set 17.C1. This value is likely an overestimation because of positive correlation (Fig. 3D) between observations in data set 17.C1 (12).

Cyclic samples: Autocorrelation analysis. Sample ACFs for the seven cyclic samples are shown in Figure 3. In five of the seven samples, autocorrelation analysis led to the identification of disease patterns not detected in total-census samples. Sample ACFs from cyclic samples 14.C1 (Fig. 3A), 16.C1 (Fig. 3C), and 17.C2 (Fig. 3E) had more values of $r(s)$ outside the $95 \%$ CI than would be expected due to random chance. An ARIMA(l 000$)$ model did not provide an adequate description of the disease in these samples. Also, sample ACFs for data sets 14.C1 (Fig. 3A), 16.C1 (Fig. 3C), and 17.C2 (Fig. 3E) appeared sinusoidal. In all three data sets, this pattern had a wavelength of $\sim 400$ plants. The sample ACF from cyclic sample 19.C1 (Fig. 3B1) exhibited an overall linear decrease in $r(s)$ as $s$ increased, consistent with the ARIMA(llll $\left.\begin{array}{ll}0 & 1\end{array}\right)$ model $\left(\hat{\theta}_{1}=0.8747\right)$ fit to total-census data collected with this cyclic sample. The total number of $r(s)$ values outside the $95 \% \mathrm{CI}$ was well within the number expected due to random chance, but an abnormally high number of $r(s)$ values fell outside the lower confidence band. For cyclic sample 17.C1 (Fig. 3D), the number of values of $r(s)$ outside the CI also was well within the number expected due to random chance. However, a series of $r(s)$ values for small $s$ (i.e., $s \leq 10$ ) was outside the $95 \% \mathrm{CI}$ and was uncharacteristic of the ARIMA(0 000$)$ model from which the 95\% CI for this cyclic sample was derived. This sample ACF also had a slight sinusoidal appearance.

Only sample ACFs from cyclic samples 21.C1 (Fig. 3F) and 21.C2 (Fig. 3G) showed no evidence of additional disease patterns compared to their corresponding total-census samples. The sample ACF from cyclic sample 21.C1 (Fig. 3F) had an initial exponential decrease in $r(s)$ values characteristic of the ARIMA(llll 00 ) model fit to total-census data collected in conjunction with this cyclic sample. The $r(s)$ values for cyclic sample 21.C2 (Fig. 3G) were also well within the corresponding CI.

Cyclic samples: Spectral analysis. $R^{2}$ values obtained from spectral analyses of the seven cyclic samples are plotted in Figure 4. All seven spectral profiles had at least one $R^{2}$ peak outside the corresponding liberal CI. Peaks occurred at short $(<200)$ and long $(\geq 200)$ wavelengths. Short wavelength peaks did not appear to occur at any one wavelength or group of wavelengths across data sets and were never outside conservative CIs. However, two of the seven cyclic samples (14.C1, and 17.C2) had long wavelength peaks in $R^{2}$ that extended beyond conservative CIs (Fig. 4A and $\mathrm{E}$, respectively). In sample 14.C1, the strongest $R^{2}$ peak occurred at a wavelength of 419 plants (Fig. 4A). Similarly, in sample 17.C2, a peak in $R^{2}$ occurred at a wavelength of 507 plants (Fig.
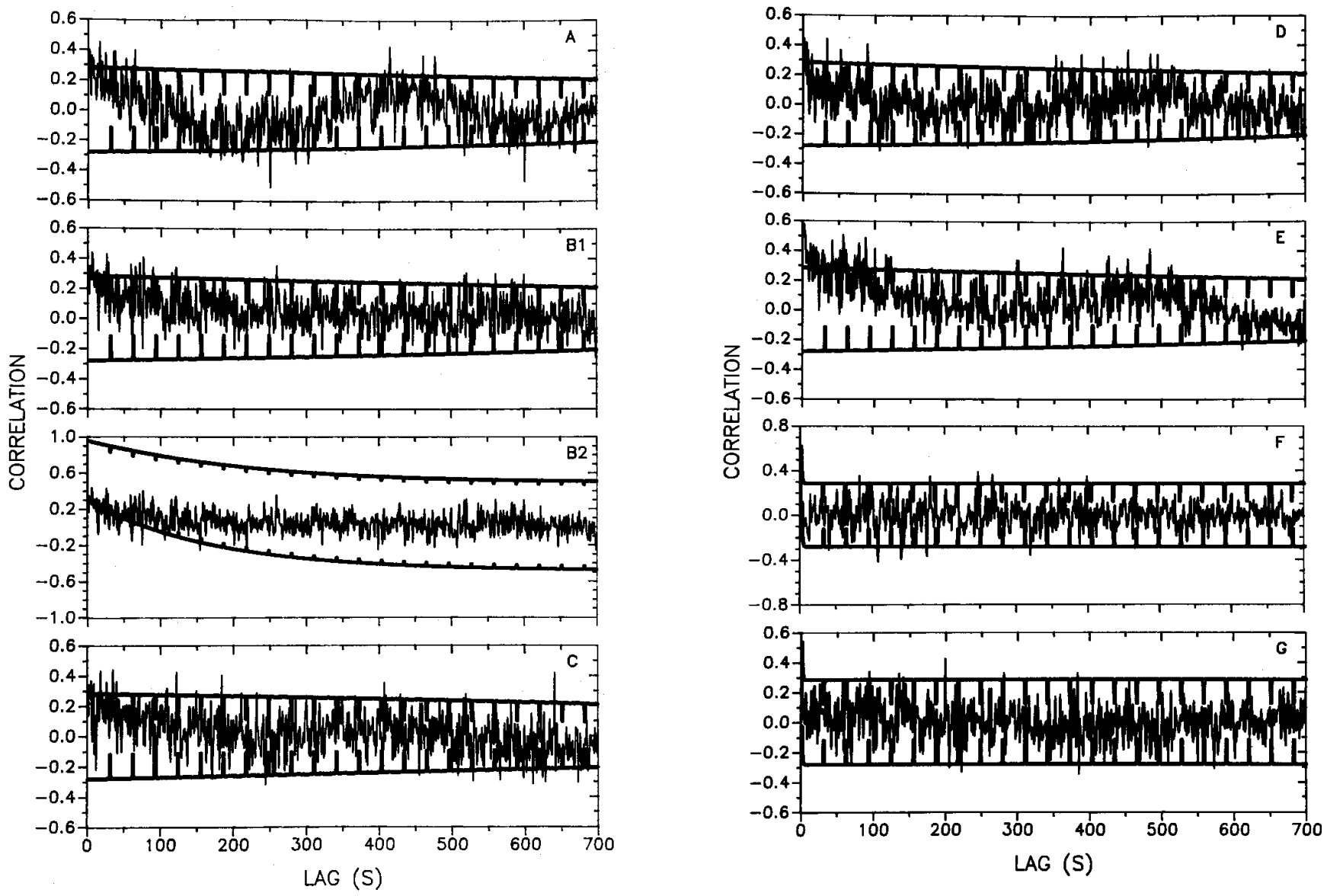

Fig. 3. Autocorrelation functions (ACFs) calculated from cyclic sample data (-) for bacterial brown spot of snap bean with corresponding $95 \%$ confidence intervals (CIs) (-). ACFs and CIs were calculated based on the results of Clayton and Hudelson (6). Spikes (|) in the CIs occur at lag values that are multiples of 31-the length of the repeating unit in the cyclic sample used to collect the data in this study (Fig. 1). These spikes reflect the increased number of pairs of plants available for calculating the correlation at these lag values compared to other lag values (6). A, ACF from data set 14.C1; 95\% CIs are based on the ARIMA( $\left(\begin{array}{lll}0 & 0\end{array}\right)$ model fit to total-census data set 14.2. B1, ACF from data set 19.C1; $95 \%$ CIs are based on an ARIMA(0 0 0) model. B2, ACF from data set 19.C1; 95\% CIs are based on an ARIMA(1 01$)$ model $\left(\phi_{1}=0.995\right.$ and $\left.\theta_{1}=0.876\right)$ fit to total-census data set 19.1. C, ACF from data set $16 . \mathrm{C} 1 ; 95 \%$ CIs are based on the ARIMA( $\left(\begin{array}{lll}0 & 0\end{array}\right)$ models fit to total-census data sets 16.1 and 16.2. D, ACF from data set 17.C1; 95\% CIs are based on the ARIMA( 000$)$ model fit to total-census data set 17.2. E, ACF from data set 17.C2; 95\% CIs are based on the ARIMA(0 00 ) model fit to total-census data set 17.2. F, ACF from data set 21.C1; $95 \%$ CIs are based on the ARIMA $\left(\begin{array}{lll}1 & 0 & 0\end{array}\right)$ model $\left(\phi_{1}=0.340\right)$ fit to total-census data set 21.1. G, ACF from data set $21 . \mathrm{C} 2$; $95 \%$ CIs are based on the ARIMA(1 00$)$ model $\left(\phi_{1}=0.260\right)$ fit to total-census data set 21.2. 
4E). Peaks in $R^{2}$ outside the liberal but not the conservative CIs occurred at similar wavelengths in samples 16.C1 (509 plants), 17.C1 (502 plants), and 19.C1 (567 plants) (Fig. 4C, D, and B1, respectively). The physical distances at which these patterns occurred (based on an estimate of the average plant density of the field from which the cyclic sample was collected) were $24.2 \mathrm{~m}$ (sample 14.C1), $35.4 \mathrm{~m}$ (sample 17.C2), $21.4 \mathrm{~m}$ (sample 16.C1), $35.0 \mathrm{~m}$ (sample 17.C1), and $38.5 \mathrm{~m}$ (sample 19.C1). In addition to this 400 to 600 plant pattern, two data sets (16.C1 [Fig. 4C] and 17.C2 [Fig. 4E]) exhibited values of $R^{2}$ that were outside conservative CIs at a wavelength of 1,550 , whereas two other data sets (17.C1 [Fig. 4D] and 19.C1 [Fig. 4B1]) exhibited values of $R^{2}$ that were outside liberal CIs at this wavelength. For data sets 16.C1 (Fig. 4C), 17.C2 (Fig. 4E), and 19.C1 (Fig. 4B1), the $R^{2}$ values associated with this wavelength were larger than for any other wavelength.

Stepwise regression selected a very limited number of wavelengths of sine and cosine curves that could be used to describe a relatively large proportion ( 22.8 to $52.3 \%$ ) of the variability in the cyclic samples (Table 4). Predicted disease values for the seven cyclic samples, calculated based on the wavelengths selected by stepwise regression, are plotted in comparison to observed disease values in Figure 2. In all seven data sets, selected wavelengths included both short ( $<200$ plants) and long ( $\geq 200$ plants) wave- lengths. Prediction models for all seven cyclic samples included wavelengths in the 6 to 23 plant range. For four data sets (14.C1 [Fig. 2A], 16.C1 [Fig. 2C1], 17.C1 [Fig. 2D1], and 21.C1 [Fig. $2 \mathrm{~F}]$ ), stepwise regression selected wavelengths in the 210 to 260 plant range. In five data sets (14.C1 [Fig. 2A], 16.C1 [Fig. 2C1], 17.C1 [Fig. 2D1], 17.C2 [Fig. 2E1], and 19.C1[Fig. 2B1]), a 400 to 600 plant wavelength was selected. In four data sets (16.C1 [Fig. 2C1], 17.C1 [Fig. 2D1], 17.C2 [Fig. 2E1], and 19.C1 [Fig. 2B1]), sine and cosine curves of wavelength 1,550 explained a relatively large proportion of disease variability. This latter variability could be explained alternatively by linear (19.C1 [Fig. 2B2]) or by linear and quadratic (16.C1 [Fig. 2C2], 17.C1 [Fig. 2D2], and 17.C2 [Fig. 2E2]) trends. Wavelengths of other sine and cosine curves included in the models were, in general, the same when sine and cosine curves of wavelength 1,550 or linear or quadratic trends were used. However, the order in which particular wavelengths were selected was sometimes altered.

\section{DISCUSSION}

In this study, using cyclic samples and associated analytical tools, we have been able to detect and describe patterns of bacterial brown spot at several different scales within a given single snap
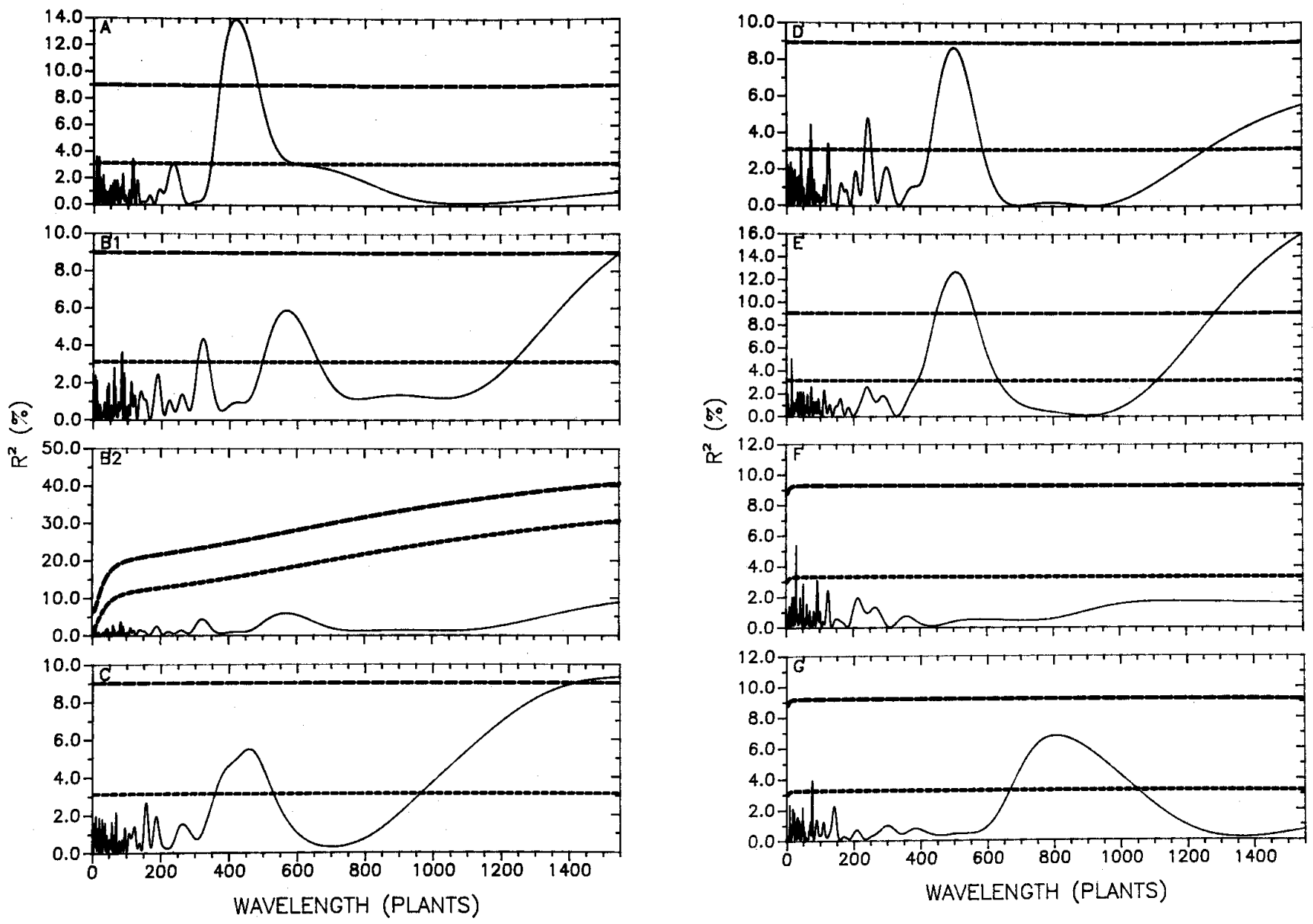

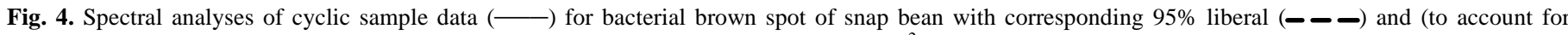

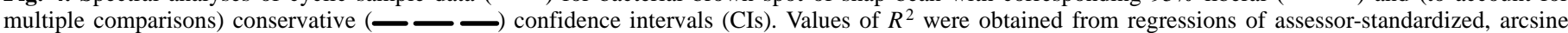

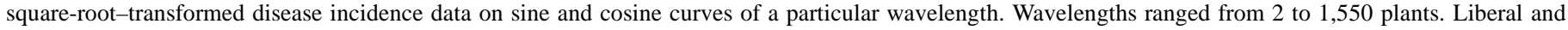

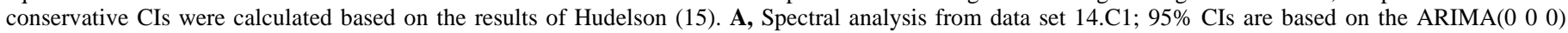

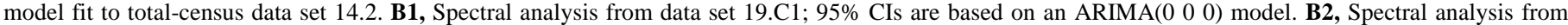

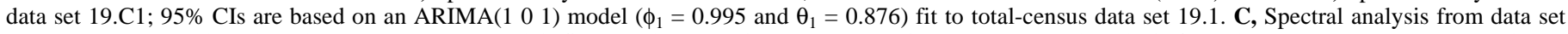

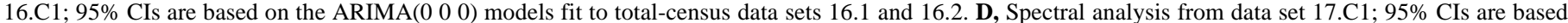

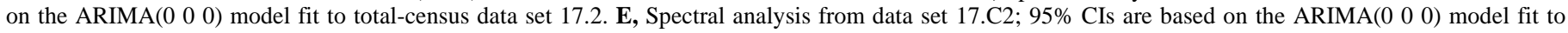

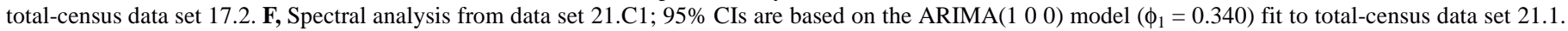
G, Spectral analysis from data set 21.C2; $95 \%$ CIs are based on the ARIMA(1 000$)$ model $\left(\phi_{1}=0.260\right)$ fit to total-census data set 21.2 . 
bean row. These patterns occurred at distances that ranged from $\sim 20$ to $\sim 100 \mathrm{~m}$ in length. We also observed patterns that occurred at short distances similar to those observed with other sampling techniques (e.g., 2,4-systematic samples [17]). We have reason to believe that some disease patterns can be stable over time (Fig. $4 \mathrm{D}$ and $\mathrm{E}$ ), although changes in the relative magnitude of patterns over time can occur. Within adjacent rows in the same field (samples 21.C1 and 21.C2), consistency in disease patterns is less clear. Peaks in $R^{2}$ seemed to occur at different wavelengths, but peaks in both data sets were relatively small, with only one (sample 21.C1) or two (sample 21.C2) extending beyond the most liberal confidence band (Fig. 4F and G, respectively). Neither sample exhibited strong nonrandom disease patterns. Based on our limited sampling, conclusions about the consistency of patterns over time and across row are difficult to make. Additional work to characterize temporal changes in disease patterns and pattern similarities or differences across rows is needed.

The intriguing characteristic of cyclic sample disease patterns is not necessarily their presence but, instead, their regularity. In all seven cyclic samples, $5 \%$ or more of the variability in the data could be described by sine and cosine curves of a single wavelength. This percent, although small in absolute magnitude, was much larger than would be expected if there were no nonrandom disease patterns or only those patterns detected within total-census data sets. In addition, for all of the seven cyclic samples we collected, more than $20 \%$ of the variability in disease could be explained by a small number of sine and cosine curves (more than $50 \%$ for cyclic sample 17.C1). Thus, knowledge of disease spatial patterns appears to be important in understanding the overall variability in plant disease epidemics.

Sine and cosine curves undoubtedly provide an artificial picture of bacterial brown spot patterns. However, the regularity suggested by sine and cosine curves may indicate that the underlying mechanism leading to these patterns has some regular, repeating component. A prime area to explore for mechanistic explanations of these patterns would be human activity in snap bean fields. These activities can be numerous and varied, including plowing, discing, harrowing, fertilizer or pesticide application (incorporated

TABLE 4. Results from spectral and stepwise regression analyses of bacterial brown spot incidence on snap beans collected using cyclic samples

\begin{tabular}{llc}
\hline Data set $^{\mathrm{a}}$ & \multicolumn{1}{c}{ Selected wavelengths $^{\mathrm{b}}$} & $\begin{array}{c}\text { Percent variability } \\
\text { explained }\left(R^{2}\right)\end{array}$ \\
\hline 14.C1 & $419,655,9,116,224,57,19,86$ & 36.1 \\
$16 . \mathrm{C} 1$ & $1,550,422,157,591,10,259,70$ & 26.3 \\
& $\mathrm{~L}^{\mathrm{c}}, \mathrm{Q}^{\mathrm{d}}, 413,697,157,10,70,249$ & 24.8 \\
$17 . \mathrm{C} 1$ & $502,1,550,73,246,43,125,13,45$, & 36.1 \\
& $25,75,450$ & \\
& $\mathrm{~L}, \mathrm{Q}, 533,73,43,243,125,13,45$, & 35.2 \\
& 76,25 \\
$17 . \mathrm{C} 2$ & $1,550,539,478,39,74,50,304,3$, & 52.3 \\
& $62,43,88,21,15,159$ & \\
& $\mathrm{~L}, \mathrm{Q}, 533,666,74,39,50,43,333$, & \\
$19 . \mathrm{C} 1$ & $62,3,21,88,15$ & \\
& $1,550,588,318,83,61,6,1,549,45$, & \\
& $112,42,19,1,548,40$ & 39.4 \\
$21 . C 1$ & $\mathrm{~L}, 673,323,83,61,6,45,12,42,19$, & \\
$21 . \mathrm{C} 2$ & $40 \quad 93,51,125,42,24,211,19$ & 22.8 \\
\hline
\end{tabular}

a Numbers to the left of the decimal point refer to the field from which cyclic and total-census samples were collected. The use of the letter $\mathrm{C}$ plus a number to the right of the decimal point denotes a cyclic sample. Data sets 17.C1 and 17.C2 were collected from the same row segment on two different days. Data sets 21.C1 and 21.C2 were collected from adjacent rows in the same field.

${ }^{\mathrm{b}}$ Wavelengths are listed in descending order of the magnitude of their contribution to the percent variability explained.

c A linear trend was included in the model.

${ }^{\mathrm{d}}$ A quadratic trend was included in the model. in soil or by air), irrigation, tilling, harvesting, etc. Some of these practices may occur at an angle relative to the row direction and, thus, potentially could be associated with disease patterns at distances anywhere between $0 \mathrm{~m}$ (i.e., the practice occurred parallel to the row direction) and the width of the equipment used in the practice (i.e., the practice occurred perpendicular to the row direction). In addition, the possible carry-over effects from activities performed on previous crops grown in the same field must be considered.

One possible correspondence between an observed disease pattern and human activity occurred in cyclic sample 14.C1, in which a disease pattern present at a distance of approximately $38 \mathrm{~m}(655$ plants) closely approximated the width of the equipment $(36.5 \mathrm{~m})$ used to spray fungicides on potatoes grown in the field during the previous year, 1987. Although direct links between fungicide applications in 1987 and the subsequent bacterial brown spot disease patterns we observed are impossible to make (e.g., we were unable to verify whether fungicide applications to potatoes in 1987 were made perpendicular to the snap bean row direction in 1988), this correspondence provides incentives to investigate the role of pesticides in disease spatial-pattern development in future field experiments.

Although some of the disease patterns we observed may have had a human origin, nonhuman origins also must be considered. For example, disease patterns that occur at distances of 400 to 600 plants were present in data sets 14.C1, 16.C1, 17.C1, 17.C2, and 19.C1. A single mechanism or very similar mechanisms may have been involved in the development of these patterns (although different mechanisms also are possible). One scenario that might account for the similarity in disease patterns in the 400 to 600 plant range is that they may be related to the relative frequency with which $P$. syringae pv. syringae-infested seed occurred in the seed lots used to plant these fields. Given adequate mixing of the bean seeds in the hoppers of the planter, seeds should be randomly distributed within a row, and the distance (in number of plants) between infested seeds in the row should be distributed as a geometric random variable with parameter $p$, where $p$ is the frequency with which infested seeds occur in the seed lot.

For a geometric distribution, the frequency with which a particular distance between infested seeds occurs would not be equal for all distances. Given localized development of disease surrounding the location of the infested seeds, one might expect to see a peak in $R^{2}$ plots related to the seed infestation frequency. Results from other work in which the locations of bacterial brown spot patches within a 4-ha snap bean field were monitored (15) are consistent with this hypothesis. The median distance (in plants) between disease patches (which could potentially have arisen as the result of infested seed) was approximately 434 to 453 plants. Interestingly, this is similar to the 400 plant pattern detected by cyclic sampling. One can hypothesize that the 600 plant pattern detected by cyclic sampling may have arisen through a similar mechanism, but with a different initial level of infested seed. The possible impact of seed infestation on disease pattern development might be investigated further through simulation studies or field testing of seed lots with different levels of infestation.

In addition to disease patterns occurring in the range of 400 to 600 plants, several cyclic samples have high $R^{2}$ values at plant distances of 1,550 plants $(60$ to $100 \mathrm{~m})$. High $R^{2}$ values at this distance may indicate the presence of additional longer wavelength sinusoidal patterns or may indicate that noncyclic changes in the mean level of disease are occurring in the row segments. Use of sine/cosine curves versus linear/quadratic trends yielded similar results when cyclic samples were modeled (Table 4). Collection of cyclic samples from longer row segments will be necessary to clarify the nature and hopefully provide insights into the origin of these patterns. Such samples also should provide information on even larger scale brown spot spatial patterns. Given the currently identified complexity of spa- 
tial patterns in the brown spot system, the presence of additional patterns within rows seems likely.

In our current work, we concentrated on describing bacterial brown spot patterns within rows. We chose a unidimensional approach because the techniques for such an approach (including cyclic sampling, ARIMA modeling, and spectral analysis) are well developed, well documented, and easily accessible to plant pathologists. This approach allowed the identification of several disease patterns and has been useful in developing possible mechanistic explanations for the patterns that can be tested in future field studies. Disease development, however, is a multidimensional process, and this study generated only limited information on patterns across rows (data sets 21.C1 and 21.C2) and through time (data sets 17.C1 and 17.C2). There likely are patterns of bacterial brown spot across rows and interactions between within and across row patterns that we failed to detect because of our unidimensional approach.

An ideal strategy for studying disease spatial patterns would be one that allows detection and description of patterns in many dimensions simultaneously. However, collecting, analyzing, and interpreting multidimensional disease data is extremely difficult. Multidimensional tools equivalent to those we used in our unidimensional studies are not available. A multidimensional version of ARIMA modeling, called STARIMA modeling, has been described previously in the plant pathology literature $(24,25)$. However, parameter estimation for these and related models is difficult and limited to a small subset of models (1). Also, efficient sampling techniques for collecting multidimensional data are not well defined. An efficient sampling technique would minimize the number of plants that need to be sampled while retaining information on disease patterns in all dimensions. We are currently working to develop multidimensional cyclic samples that will fulfill these criteria. Only with further development of modeling tools and sampling techniques will a more detailed and complete picture of the complexity of spatial and temporal patterns of plant diseases, including bacterial brown spot of snap bean, be possible.

\section{ACKNOWLEDGMENTS}

Cooperative research of the College of Agriculture and Life Sciences, University of Wisconsin, Madison, and the Agricultural Research Service, U.S. Department of Agriculture. Research supported in part by USDA CSRES Competitive Grant 88-37151-3898. We thank K. Hoffman and J. Armstrong for valuable technical assistance and S. Vicen for preparation of the figures. We also thank the snap bean growers and food processors of Wisconsin for their cooperation in providing commercial snap bean fields for this work.

\section{LITERATURE CITED}

1. Basu, S., and Reinsel, G. 1993. Properties of the spatial unilateral firstorder ARMA model. Adv. Appl. Probab. 25:631-648.

2. Box, G. E. P., Jenkins, G. M., and Reinsel, G. C. 1994. Time Series Analysis: Forecasting and Control. 3rd ed. Prentice Hall, Englewood Cliffs, NJ.

3. Brockwell, P. J., and Davis, R. A. 1987. Time Series: Theory and Methods. Springer-Verlag, New York.

4. Chatfield, C. 1984. The Analysis of Time Series: An Introduction. 3rd ed. Chapman and Hall, New York.

5. Chellemi, D. O., Rohrbach, K. G., Yost, R. S., and Sonoda, R. M. 1988. Analysis of the spatial pattern of plant pathogens and diseased plants using geostatistics. Phytopathology 78:221-226.

6. Clayton, M. K., and Hudelson, B. D. 1995. Confidence intervals for autocorrelations based on cyclic samples. J. Am. Stat. Assoc. 90:753-757.

7. Clinger, W., and Van Ness, J. W. 1976. On unequally spaced time points in time series. Ann. Stat. 4:736-745.

8. Cressie, N. A. C. 1991. Statistics for Spatial Data. John Wiley \& Sons, New York.

9. Cryer, J. D. 1986. Time Series Analysis. Duxbury Press, Boston.

10. Diggle, P. J. 1979. On parameter estimation and goodness-of-fit testing for spatial point patterns. Biometrics 35:87-101.

11. Diggle, P. J., and Cox, T. F. 1983. Some distance-based tests of independence for sparsely-sampled multivariate spatial point patterns. Int. Stat. Rev. 51:11-23.

12. Draper, N. R., and Smith, H. 1981. Applied Regression Analysis. 2nd ed. John Wiley \& Sons, New York.

13. Freund, J. E. 1981. Statistics: A First Course. 3rd ed. Prentice Hall, Englewood Cliffs, NJ.

14. Gray, S. M., Moyer, J. W., and Bloomfield, P. 1986. Two-dimensional distance class model for quantitative description of virus-infected plant distribution lattices. Phytopathology 76:243-248.

15. Hudelson, B. D. 1990. Studies of the Spatial Patterns of Bacterial Brown Spot of Snap Beans. Ph.D. thesis. University of Wisconsin, Madison.

16. Hudelson, B. D., Clayton, M. K., Smith, K. P., Rouse, D. I., and Upper, C. D. 1989. Nonrandom patterns of bacterial brown spot in snap bean row segments. Phytopathology 79:674-681.

17. Hudelson, B. D., Clayton, M. K., Smith, K. P., and Upper, C. D. 1993. Modeling of superimposed spatial patterns of bacterial brown spot of snap bean. Phytopathology 83:430-438.

18. Lecoustre, R., Fargette, D., Fauquet, C., and de Reffye, P. 1989. Analysis and mapping of the spatial spread of African cassava mosaic virus using geostatistics and the kriging technique. Phytopathology 79:913-920.

19. Mihail, J. D., and Alcorn, S. M. 1987. Macrophomina phaseolina: Spatial patterns in a cultivated soil and sampling strategies. Phytopathology 77:1126-1131

20. Munkvold, G. P., Duthie, J. A., and Marois, J. J. 1993. Spatial patterns of grapevines with Eutypa dieback in vineyards with or without perithecia. Phytopathology 83:1440-1448.

21. Nicot, P. C. 1985. Epidemiology of Verticillium wilt of potato: Spatial patterns of soilborne Verticillium dahliae and incidence of stem colonization. Ph.D. thesis. University of Wisconsin, Madison.

22. Poushinsky, G., and Basu, P. K. 1984. A study of distribution and sampling of soybean plants infected with Pseudomonas syringae pv. glycinea. Phytopathology 74:319-326.

23. Proctor, C. H. 1984. On the detection of clustering and anisotrophy using binary data from a lattice patch. Commun. Stat.-Theory Methods 13: 617-638.

24. Reynolds, K. M., and Madden, L. V. 1988. Analysis of epidemics using spatio-temporal autocorrelation. Phytopathology 78:240-246.

25. Reynolds, K. M., Madden, L. V., and Ellis, M. A. 1988. Spatio-temporal analysis of epidemic development of leather rot of strawberry. Phytopathology 78:246-252.

26. Ripley, B. D. 1981. Spatial Statistics. John Wiley \& Sons, New York.

27. Ristaino, J. B., Larkin, R. P., and Campbell, C. L. 1993. Spatial dynamics of disease symptom expression during Phytophthora epidemics in bell pepper. Phytopathology 84:1015-1024.

28. Ristaino, J. B., Larkin, R. P., and Campbell, C. L. 1994. Temporal and spatial dynamics of Phytophthora epidemics in commercial bell pepper fields. Phytopathology 83:1312-1320.

29. Seber, G. A. F. 1984. Multivariate Observations. John Wiley \& Sons, New York.

30. Snedecor, G. W., and Cochran, W. G. 1989. Statistical Methods. 8th ed. Iowa State University Press, Ames.

31. Thal, W. M., and Campbell, C. L. 1986. Spatial pattern analysis of disease severity data for alfalfa leaf spot caused primarily by Leptosphaerulina briosiana. Phytopathology 76:190-194.

32. Upton, G., and Fingleton, B. 1985. Spatial Data Analysis by Example. John Wiley \& Sons, Chichester, England. 